\title{
Differential expression of keratins 10, 17, and 19 in normal cervical epithelium, cervical intraepithelial neoplasia, and cervical carcinoma
}

\author{
Peter Maddox, Peter Sasieni, Anne Szarewski, Malcolm Anderson, Andrew Hanby
}

\begin{abstract}
Aim-To examine the value of immunohistochemistry in defining a keratin profile to aid cervical histopathological diagnosis.

Methods-Immunohistochemical localisation of keratins 17,10 , and 19 was studied in 268 cervical biopsies from 216 women including normal epithelia (with and without human papilloma virus), low and high grade cervical intraepithelial neoplasia, and invasive carcinoma. The percentage of positive immunostaining was scored using a Kontron MOP videoplan image analyser.

Results-All major categories of cervical epithelia expressed these keratins to varying degrees. The median percentage of immunostaining for keratin 10 was $40 \%$ in normal tissue compared with just $1 \%$ in invasive carcinoma $(p<0.0001)$. The medians for keratin 17 were $0 \%$ in the normal group and $80 \%$ in carcinomas $(p<0.0001)$. By contrast, there was no significant difference in staining for keratin 19. Using a combination of the keratin 10 and 17 percentages, it was possible to separate the carcinomas from the benign conditions with a sensitivity of $100 \%$ and a specificity of $93 \%$. Further analyses within the groups revealed more extensive staining for keratins 10 and 19 in reserve cell hyperplasia, immature squamous metaplasia, and congenital transformation zone.

Conclusions-The morphological variety within the cervix is reflected, in part, by distinct keratin patterns. There are striking differences in the patterns of keratins 10 and 17 between infiltrating squamous carcinoma and normal cervical epithelia. (F Clin Pathol 1999;52:41-46)
\end{abstract}

Keywords: keratin expression; cervical pathology; CIN

Histopathology,

Queen's Medical

Centre, Nottingham,

UK

M Anderson

ICRF Histopathology

Unit and Clinical

Oncology Unit, Guys'

Hospital, London SE1, UK

A Hanby

Correspondence to: Dr Sasieni.

Accepted for publication 14 September 1998

The cervix is host to a bewildering array of morphological variations including not only a range of premalignant and malignant alterations, but also harmless changes that may mimic those of more serious lesions. Previous studies have indicated that there are distinct patterns of keratin expression associated with the different morphological states. ${ }^{1}$ These studies have generally only examined a small number of samples and have described the keratin staining qualitatively, not quantitatively. In this study we set out to describe variations in keratin staining quantitatively in a relatively large number of cervical samples from different women.

The commonest variation from normal is squamous metaplasia, which in the cervix is physiological and considered a normal event. ${ }^{2}$ This process leads to replacement of the delicate thin layer of columnar cells lining the cervix with a thicker, stronger, and more protective layer of squamous cells. In mature squamous metaplasia there is a complete absence of residual columnar cells, while the epithelium becomes fully differentiated on its surface and of similar thickness to the original squamous epithelium. This alteration is readily interpretable. However, there are variants which may pose more interpretative challenges. In reserve cell hyperplasia, a layer of cells with morphological attributes of the basal squamous epithelium proliferates just beneath the columnar epithelial cells; this process, in its most advanced form, may lead to a layer several cells thick, resulting in some fusion of the endocervical villi. Immature squamous metaplasia is the result of further differentiation and maturation of reserve cells, with larger nuclei, prominent nucleoli, and a relative increase in cytoplasm. Epithelial stratification is not apparent at this stage, although residual groups of mucin containing columnar cells may be seen above or within the immature squamous layer. Owing to the marked histological similarity to squamous metaplasia, the expression "congenital transformation zone" is not always recognised by pathologists as a variant. However, typically it shows an irregular maturation often delayed in deeper layers and advanced in the upper layers of the epithelium resulting in hyperkeratosis, parakeratosis, and sometimes leucoplakia. The most striking histological feature is the irregular dentate appearance at the epithelial-stromal junction which can, if cross cut, appear separated from the overlying epithelium and give a false impression of an invasive lesion.

There are at least 20 intermediate filament proteins which are distributed throughout the epithelia, ${ }^{4}$ subdivided into acidic (type I) and basic (type II) subgroups, each the product of a distinct keratin gene. These are expressed in a tissue specific manner, with a tendency to be coexpressed as obligate pairs. The availability of commercial antisera to individual keratin polypeptides has made it possible to study the keratin phenotypes of normal epithelia and observe changes in keratin expression patterns of both metaplastic epithelia and epithelia with 
Table 1 Summary of the antibodies employed in the study and their modes of use

\begin{tabular}{lllll}
\hline Keratin & Antibodies & $\begin{array}{l}\text { Microwave } \\
\text { (time) }\end{array}$ & $\begin{array}{l}\text { Incubation overnight } \\
\text { (dilution) }\end{array}$ & $\begin{array}{l}\text { Incubation } 1 \mathrm{~h} \\
\text { (dilution) }\end{array}$ \\
\hline 10 & Dako M7002 DE-K10 & Unnecessary & $1: 200$ & $1: 50$ \\
19 & Dako M888 & 10 min & $1: 80$ & $1: 50$ \\
17 & Dako M7046 & $10 \mathrm{~min}$ & $1: 70$ & $1: 10$ \\
\hline
\end{tabular}

malignant potential. ${ }^{5}$ Previous studies have indicated that both benign and malignant cervical epithelia may show distinct morphology related patterns of keratin expression..$^{6-9}$ Keratins 10,17 , and 19 are of particular interest.

Keratin 10 (K10) is a suprabasal differentiation related keratin restricted to skin and cervix and has been associated with acetowhite change, ${ }^{6}$ whereby application of acetic acid to the cervix at colposcopy reveals some abnormalities as distinct white patches. This is rare in completely normal cervices. It is associated with keratinisation although no signs of keratinisation have so far been detected in the normal cervix. ${ }^{7}$ Keratin 17 (K17) was first identified within the pilosebaceous tract and basal cell carcinoma ${ }^{4}$ but subsequently has been seen in malignantly transformed epidermis. ${ }^{10}$ It has also been suggested as a useful marker for distinguishing carcinoma of the cervix from mesotheliomas. ${ }^{9}$

Keratin 19 (K19) is expressed as an intermediate type 1 keratin and lacks the carboxyl terminal non-helical domain which suggests a flexibility of differentiation, ${ }^{11}$ possibly allowing delayed synthesis of the specific type 1 keratin. It is found in the full thickness of metaplastic cervical epithelium, but only in the basal layer of normal epithelium. ${ }^{12}$

Since the interpretation of the disparate histological appearances seen within the cervix, from metaplasia to cervical intraepithelial neo- plasia (CIN), suffers considerable interobserver variation, we have examined the practical value of immunohistochemistry in defining a keratin profile to aid diagnosis.

\section{Methods}

MATERIALS

Tissue in the form of 268 specimens from 216 patients was collected; $7 \%$ of these were from diathermy loop excisions, $24 \%$ from cone biopsies, $43 \%$ from colposcopic biopsies, and $25 \%$ from hysterectomies. The cone biopsies yielded frozen sections, the rest were paraffin blocks. The carcinoma specimens were accrued from 36 women with cervical carcinoma. The histology of all 268 samples was reviewed for this study.

\section{HISTOLOGICAL PROCEDURES}

Formalin fixed, parallel paraffin sections were taken at $5 \mu \mathrm{m}$ intervals and stained with haematoxylin and eosin (H\&E) and with one of the three listed antibodies to keratins 10, 17, and 19 (table 1). For two of the antibodies, sections were treated in a microwave oven at $700 \mathrm{~W}$ in sodium citrate buffer, $\mathrm{pH} 6$, and then cooled rapidly under running water before immunostaining. ${ }^{13}$ Endogenous peroxidase activity was inhibited by using $0.5 \%$ hydrogen peroxide for 10 minutes. After incubation for the times indicated in table 1 , biotinylated rabbit antimouse immunoglobulin was applied, followed by an avidin-biotin peroxidase conjugate label demonstrated with a standard 3,3 diaminobenzidine chromagen and counterstained with Mayer's haematoxylin.

Grade 1 carcinoma of the cervix refers to large cell, keratinising tumours; grade 2 to large cell, non-keratinising tumours; and grade 3 to

Table 2 Distribution of cytokeratins 10, 19, and 17 in normal, premalignant, and malignant epithelia

\begin{tabular}{|c|c|c|c|c|c|c|c|c|c|}
\hline \multirow[b]{2}{*}{ Report } & \multicolumn{3}{|l|}{ K10 } & \multicolumn{3}{|c|}{ K19 } & \multicolumn{3}{|c|}{ K17 } \\
\hline & $n$ & Median & $I Q R$ & $n$ & Median & $I Q R$ & $n$ & Median & $I Q R$ \\
\hline Normal & 112 & 43 & 10 to 80 & 74 & 22 & 8 to 44 & 55 & 0 & 0 to 3 \\
\hline LG-SIL & 40 & 41 & 19 to 77 & 27 & 10 & 6 to 50 & 20 & 0 & 0 to 0 \\
\hline HG-SIL & 34 & 17 & 3 to 60 & 20 & 30 & 6 to 65 & 8 & 0 & 0 to 19 \\
\hline Cancer & 29 & 0 & 0 to 2 & 29 & 23 & 5 to 75 & 29 & 80 & 40 to 90 \\
\hline p Value $(\mathrm{K}-\mathrm{W})$ & \multicolumn{3}{|c|}{$<0.0001$} & \multicolumn{3}{|c|}{0.51} & \multicolumn{3}{|c|}{$<0.0001$} \\
\hline $\begin{array}{l}\mathrm{p} \text { Value (trend) } \\
\text { without cancers }\end{array}$ & \multicolumn{3}{|c|}{0.058} & \multicolumn{3}{|c|}{0.68} & \multicolumn{3}{|c|}{0.88} \\
\hline
\end{tabular}

HG, high grade; IQR, interquartile range; K-W, Kruskal-Wallis; LG, low grade; SIL, squamous intraepithelial lesions.
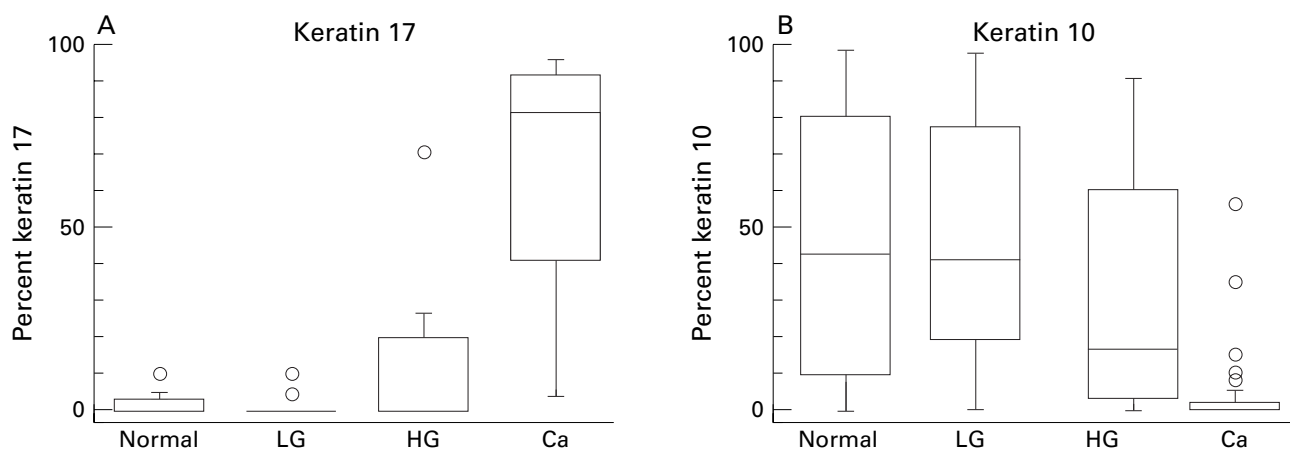

Figure 1 Box and whiskers plots of the percentage staining for cytokeratins in 55 normal, 20 low grade (LG) squamous intraepithelial lesions (SIL), eight high grade (HG) SIL, and 29 invasive cancers (Ca). (A) keratin 17; (B) keratin 10. The boxes depict the upper and lower quartiles and the median. The "whiskers" show the range with outliers marked by small circles. The median percentages of staining of keratin 17 in normal specimens, LG, and HG are all zero, which is why there are no "middle lines" in these boxes. 


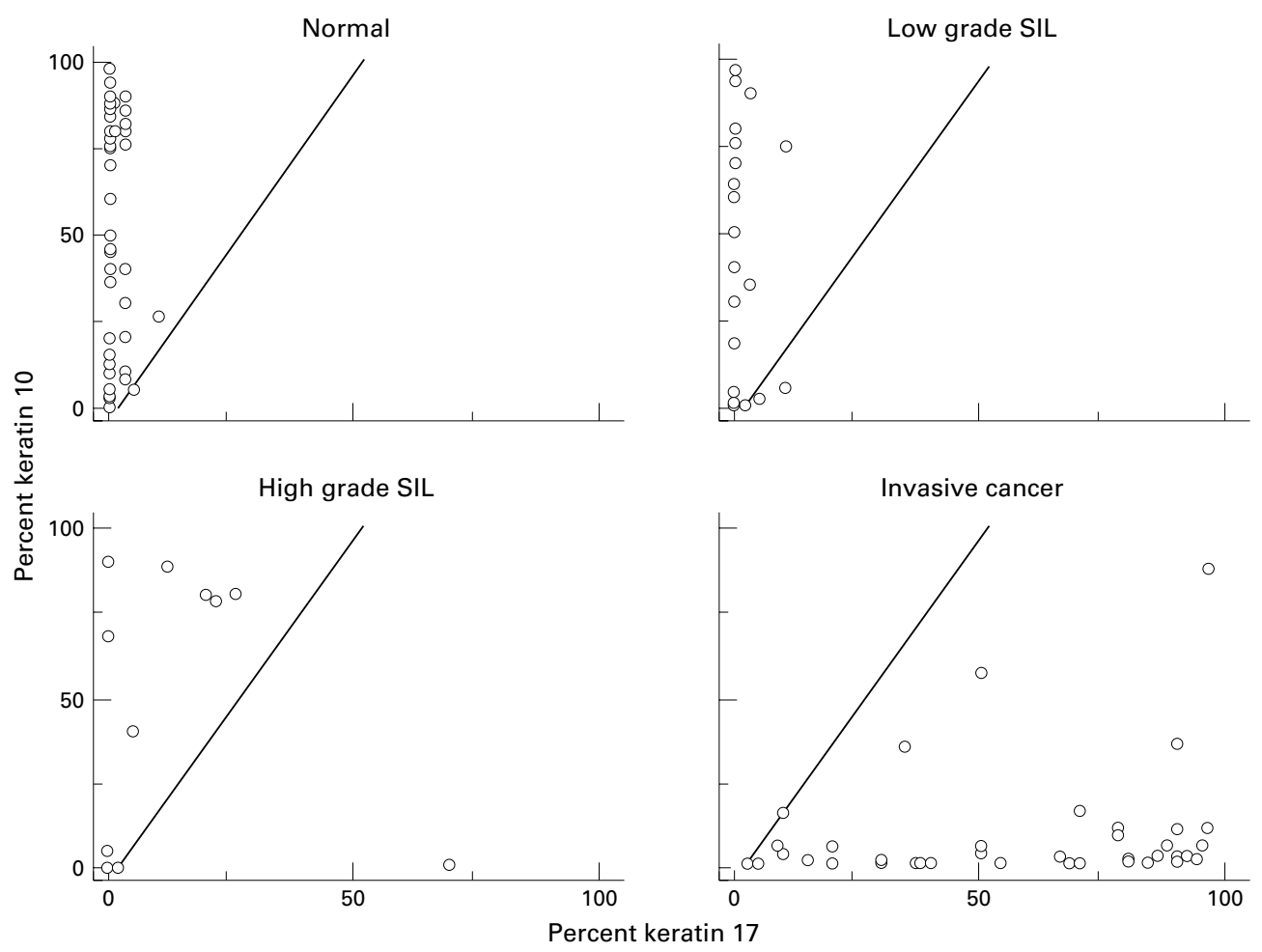

Figure 2 Scatter plots of percentage staining of keratin $10 \mathrm{v}$ keratin 17. There is a separate plot for each broad histological class. The diagonal line is the linear discriminant described in the text. Each specimen is represented by a single small circle. All specimens stained for both keratin 10 and keratin 17 are included, even though this gives multiple specimens for some women. Where two or more samples have identical keratin expression, it is not possible to distinguish their circles. Notice how keratin 10 is useful for distinguishing invasive carcinomas with low levels of keratin 17 from other samples. SIL, squamous intraepithelial lesion.

large cell, non-keratinising tumours with increasing nuclear atypia.

MORPHOLOGICAL ANALYSIS

In order to calculate the percentage of positive staining for each morphological variant, particularly where they coexisted in the same biopsy, areas of the H\&E stained and immunostained sections were marked and the percentage of positive staining in the latter calculated using a Kontron MOP videoplan image analyser. ${ }^{6}{ }^{14}$

\section{STATISTICS}

Where there was more than one specimen from the same woman, we selected (at random where necessary) a specimen with the most severe findings. Unless stated otherwise, the results relate to at most one specimen from each woman. The significance of different levels of keratin in different pathologies was assessed using the Kruskal-Wallis test. Trends in patterns of keratin expression within patho-

Table 3 Ability of linear discriminator to identify invasive carcinoma in 111 samples

\begin{tabular}{lrrr}
\hline & \multicolumn{2}{l}{ Discriminant } & \\
\cline { 2 - 4 } Report & $\leqslant 4$ & $>4$ & Total \\
\hline Normal & 54 & 1 & 55 \\
LG-SIL & 19 & 0 & 19 \\
HG-SIL & 7 & 1 & 8 \\
Cancer & 0 & 29 & 29 \\
Total & 80 & 31 & 111 \\
\hline
\end{tabular}

HG, high grade; LG, low grade; SIL, squamous intraepithelial lesions. logical subtypes were assessed using the Cuzick test for trend. ${ }^{15}$ Both of these latter methods are generalisations of the rank-sum test. Cancers were compared to each other using multiple logistic regression. ${ }^{15}$ The results presented were not altered appreciably by the use of different randomly selected specimens from the 28 women with multiple specimens.

\section{Results}

In all, 268 specimens from 216 women were used representing normal epithelia, metaplastic lesions, the low and high grades of CIN, and invasive cervical cancer. A total of 267 specimens were available for the study of keratin 10, but only 195 were stained for keratin 19, and 157 for keratin 17. The discrepancies in number were accounted for by the retrospective nature of the study rather than any form of selectivity.

\section{EXPRESSION BY GRADE OF NEOPLASIA}

Table 2 shows the median and interquartile range of the percent of tissue staining for each keratin. The expression of keratins 10 and 17 was different in the invasive carcinomas compared with the other specimens. The difference was most marked for keratin 17 . The expression of keratin 19 was not statistically different in the invasive carcinomas compared to the other samples. High grade lesions showed intermediate expression of keratins 10 and 17 , but the trends with increasing severity of non-invasive lesions were not statistically significant. The distributions of keratins 17 and 
Table 4 Distributions of cytokeratins 10,19, and 17 in non-malignant epithelia classified by type of metaplasia

\begin{tabular}{|c|c|c|c|c|c|c|c|c|c|}
\hline \multirow[b]{2}{*}{ Report } & \multicolumn{3}{|c|}{ K10 } & \multicolumn{3}{|c|}{ K19 } & \multicolumn{3}{|c|}{ K17 } \\
\hline & $n$ & Median & $I Q R$ & $n$ & Median & $I Q R$ & $n$ & Median & $I Q R$ \\
\hline $\begin{array}{l}\text { Mature } \\
\text { epithelium }\end{array}$ & 94 & 12 & 0 to 46 & 45 & 10 & 5 to 40 & 15 & 0 & 0 to 10 \\
\hline CTZ & 30 & 76 & 50 to 86 & 27 & 40 & 16 to 60 & 28 & 0 & 0 to 3 \\
\hline $\mathrm{RCH}$ & 5 & 80 & 60 to 88 & 5 & 80 & 44 to 86 & 4 & 0 & 0 to 0 \\
\hline ISM & 57 & 60 & 20 to 80 & 44 & 16 & 6 to 47 & 36 & 0 & 0 to 0 \\
\hline Kruskal-Wallis & \multicolumn{3}{|c|}{$\mathrm{p}<0.0001$} & \multicolumn{3}{|c|}{$p=0.0005$} & \multicolumn{3}{|c|}{$p=0.36$} \\
\hline
\end{tabular}

CTZ, congenital transformation zone; IQR, interquartile range; ISM, immature squamous metaplasia; RCH, reserve cell hyperplasia.

10 are illustrated in fig 1 . Using keratin 10 in addition to keratin 17 facilitated distinction of cancer specimens from the rest $(p=0.0006)$. The linear discriminant of twice the percentage of keratin 17 minus the percentage of keratin 10 effectively separated the cancers from the other specimens (fig 2). The effect of using a threshold of 4 is shown in table 3 . It gives a sensitivity of $100 \%$ for invasive carcinoma and a specificity of $93.5 \%$ in the mixture of non-malignant specimens stained for these keratins. The specificity was even better if the non-malignant specimens from women with invasive cancer were excluded, using just one specimen per woman as in table 3 . All 50 carcinoma specimens had at least 3\% staining for $\mathrm{K} 17$, whereas $71 \%$ of the other 107 specimens tested had $0 \%$ staining.

EXPRESSION BY MATURATION OF EPITHELIUM We also examined the expression of keratins in normal and benign cervical tissue categorised by congenital transformation zone (CTZ), reserve cell hyperplasia $(\mathrm{RCH})$, immature squamous metaplasia (ISM), or none of the above (mature epithelia). The results in table 4 correspond to a mixture of normal tissue and low and high grade lesions. Very similar results were obtained when restricting analysis to the normal specimens. The expression of K17, other than in invasive carcinoma, was extremely rare. Over $75 \%$ of the samples

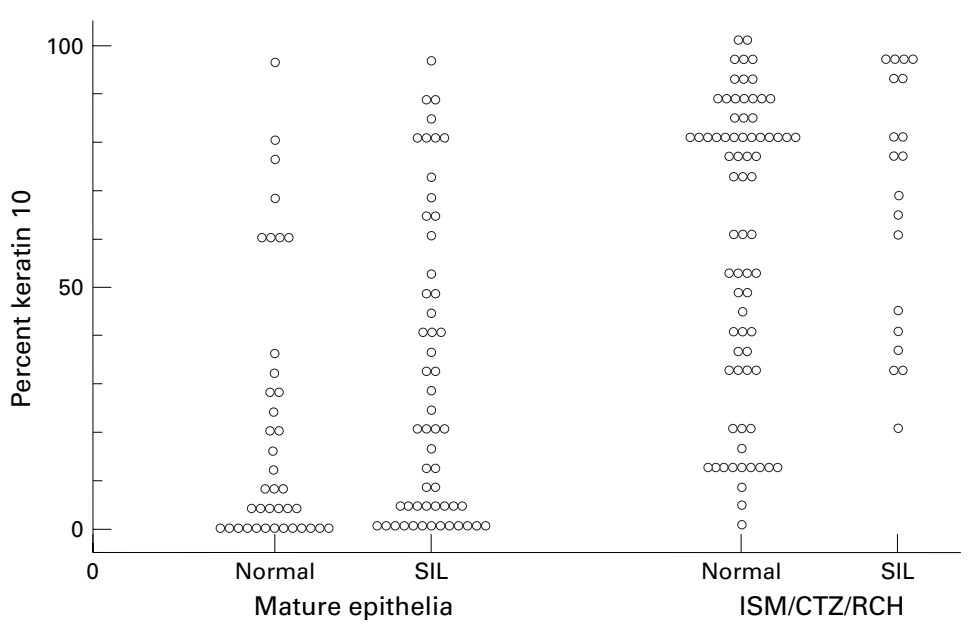

Figure 3 Distribution of percentage staining for keratin 10 by histology for non-malignant specimens. The percentage staining for keratin 10 is much greater in specimens classified as either immature squamous metaplasia (ISM), congenital transformation zone (CTZ), or reserve cell hyperplasia $(R C H)$ than in mature epithelia (that is, specimens not classified as ISM, CTZ, or RCH). It is also seen that within these two groups there is little difference in the staining for keratin 10 between normal specimens and ones showing a squamous intraepithelial lesion (SIL). showing immature squamous metaplasia had no K17 detected. Both keratin 10 and keratin 19 had higher levels of expression with CTZ, $\mathrm{RCH}$, and ISM. The weak trend of decreasing percentage of K10 staining going from normal epithelia to high grade CIN, seen in table 2, can all be accounted for by the association of CTZ, RCH, and ISM with low levels of K10 (fig 3).

STAINING IN INVASIVE CARCINOMAS

There did not appear to be any trend in the expression of keratins 17 or 19 with the grade of the invasive tumour (table 5). The expression of keratin 10 , however, decreased with grade (trend test, $\mathrm{p}=0.005$ ).

There was a significant association between focal keratosis and large cell non-keratinising (LCNK) samples and expression of K10 (but not $\mathrm{K} 17$ or $\mathrm{K} 19$ ) in the invasive tumours (data not shown). Overall $71 \%$ of the 42 non-LCNK invasive cancers expressed keratin 10, compared with none of the eight LCNK samples. The pattern was similar when restricting analysis to one specimen per woman.

\section{COMPARISON OF SPECIMENS FROM THE SAME}

WOMEN

Twenty eight women had two or more specimens in this study. Sixteen of these 28 women had invasive cancer. Four had at least one sample showing invasive cancer and one that did not. The results from these women are shown in table 6 . As can be seen, three of these four women had at least $88 \%$ staining for keratin 17 in their cancer specimens $(n=5)$ and no more than $10 \%$ in their non-cancer specimens $(n=8)$. The other woman had no more than $10 \%$ staining for keratin 17 in any of her three specimens, two of which had invasive cancer. Nevertheless, the discriminant used in table 3 would correctly distinguish between the two invasive specimens and the CIN 3 specimen in this woman. The same discriminant would incorrectly classify one sample of CIN 1 from patient 112 and one from patient 154 as "cancer." Overall, analysis of variance showed that there was considerable intra-woman correlation in keratin expression (logarithmically transformed) after adjusting for histological diagnosis. Thus, although the level of expression in different samples from a single woman varied, they were similar whenever the broad histological diagnosis was the same. 
Table 5 Expression of keratins 10,19, and 17 in invasive carcinomas

\begin{tabular}{|c|c|c|c|c|c|c|c|}
\hline \multirow[b]{2}{*}{ Report } & \multirow[b]{2}{*}{$n$} & \multicolumn{2}{|l|}{ K10 } & \multicolumn{2}{|l|}{ K19 } & \multicolumn{2}{|l|}{ K17 } \\
\hline & & Median & $I Q R$ & Median & IQR & Median & $I Q R$ \\
\hline G1 & 5 & 8.0 & 2 to 35 & 23 & 8 to 92 & 90 & 78 to 90 \\
\hline G2 & 8 & 0.5 & 0 to 2 & 14 & 2 to 63 & 74 & 15 to 87 \\
\hline G3 & 12 & 0.0 & 0 to 0 & 17 & 4 to 68 & 79 & 39 to 90 \\
\hline Not graded & 4 & 3.5 & 1 to 10 & 65 & 33 to 77 & 79 & 40 to 90 \\
\hline \multicolumn{2}{|c|}{ Trend (G1-G3) } & \multicolumn{2}{|c|}{$p=0.005$} & \multicolumn{2}{|c|}{$p=0.64$} & \multicolumn{2}{|c|}{$\mathrm{p}=0.47$} \\
\hline
\end{tabular}

Table 6 Results for four women with both benign and malignant specimens

\begin{tabular}{llccc}
\hline Patient & Histology & $\%$ K10 & $\%$ K19 & $\%$ K17 \\
\hline 112 & Inv Ca & 1 & 96 & 94 \\
& Inv Ca & 2 & 92 & 90 \\
& CIN 1 & 1 & 6 & 0 \\
& CIN 1 & 0 & 0 & 2 \\
\multirow{4}{*}{154} & CIN 1 & 5 & 8 & 10 \\
& Inv Ca & 5 & 70 & 88 \\
& CIN 1 & 2 & 50 & 55 \\
& Normal & 80 & 0 & 0 \\
180 & Normal & 0 & 9 & 0 \\
& Inv Ca & 0 & 0 & 3 \\
& Inv Ca & 3 & 30 & 10 \\
203 & CIN 3 & 0 & 30 & 2 \\
& Inv Ca & 1 & 23 & 90 \\
& Inv Ca & 10 & 60 & 90 \\
& CIN 1 & 0 & 3 & 0 \\
& CIN 1 & 0 & 0 & 0 \\
\hline
\end{tabular}

$\mathrm{CIN}$, cervical intraepithelial neoplasia; Inv $\mathrm{Ca}$, invasive carcinoma.

\section{Discussion}

The exploitation of cytokeratin antibodies for routine diagnostic pathology depends upon their expression being essentially conserved throughout extreme disruption of the tissue even into metastatic cancer. ${ }^{5}$ Since the publication of that review there have been tremendous advances in immunohistochemistry and an avalanche of commercially available antisera. However, those used for routine pathology remain few. Perhaps the explanation lies in the fact that pathologists are capable of recognising major patterns of keratin staining which correspond to keratin type by conventional histological staining methods. Within cervical pathology there is a need to improve the uniformity of tumour grade recognition and the predictability of patient survival. The availability of monospecific antibodies to individual members of the keratin family has provided our understanding of the profiles present in normal differentiating cervical squamous epithelium. The patterns in some neoplasias deviate quantitatively from that seen in normal tissue. In particular, certain profiles, such as 8 and 18, are increased in both reserve cell and cervical neoplasias, ${ }^{16}{ }^{17}$ and cytokeratins 6 and 16 are markers of hyperproliferative states. ${ }^{18}$

This study was designed as an extension of our previous publication in which we suggested that the presence of K10 in cervical epithelia may be an essential requirement for the formation of acetowhite change. This was linked with the finding that K19 profile has been associated with the pluripotential nature of stem cell populations, ${ }^{511}$ possibly similar in nature to that of $\mathrm{K} 17$, which is upregulated by gamma interferon ${ }^{19}$ and seemed to bear a sinister prognosis in terms of malignant conversion. In the case of cervical neoplasia, Smedts and colleagues identified K17 in all cervical carcino- mas examined, and in $50 \%$ of CIN 3 but in only a small proportion of CIN 1 and CIN 2 lesions. ${ }^{9}$ In 1993, Proby and her colleagues investigated the use of 20 monoclonal antibodies to 12 epithelial cytokeratins on biopsies of benign and dysplastic warts and found early expression of $\mathrm{K} 17$ and an abnormal expression of K19 in all dysplastic lesions examined. This led her to postulate that the expression of K17 may prove to be a helpful marker of early malignant change within cutaneous lesions before histological evidence of invasion. ${ }^{10}$

In this study we have provided evidence that supports the suggestion of Smedts et al that the expression of $\mathrm{K} 17$ in cervical epithelia could be considered as a prognostic marker of severe dysplasia. In addition we have shown that K10 is expressed to some degree in most hyperplastic cervical epithelia, particularly in regions of congenital transformation zone and reserve cell hyperplasia, while the expression of K10 among the carcinoma group is seen mainly among the well differentiated keratinising tumours. There is an inconsistent expression of K19 in all groups, which supports the suggestion of flexibility of function for this keratin. The observation that $\mathrm{K} 10$ expression is greatly increased in metaplastic cells (ISM, CTZ, and $\mathrm{RCH})$ together with our previous finding that K10 expression correlates with acetowhite change may explain why cervicography ${ }^{20}$ and even colposcopy are not very specific for high grade CIN, although iodine staining may better reflect the degree of neoplasia on colposcopy. Overreliance on acetowhite change may identify areas of immature squamous metaplasia and congenital transformation zone that are not associated with CIN.

It is perhaps surprising that $\mathrm{K} 10$ is present at such low levels in keratinising epithelia. It is possible that the keratinisation is caused by the presence of other similar keratins such as K11, $\mathrm{K} 9, \mathrm{~K} 1$, and $\mathrm{K} 2$. Unfortunately markers for these keratins were not available when this study was in progress. It is noteworthy that K10 expression was considerably more common in keratinising carcinomas than in LCNK cancers, even though this expression was less than in immature squamous metaplasia. Further studies looking at this group of keratins will be of considerable interest.

One might be concerned about possible epitope masking, especially because we used only one monoclonal antibody for each keratin. The very different staining patterns observed between normal and cancer tissue (for keratins 10 and 17) and between mature and immature squamous epithelium (for keratin 19) show that this was not in fact a problem with the antibodies used in our study. There remains 
the possibility that the degree of masking is dependent on the pathology. Thus, for example, neoplastic transformation could cause structural alterations in the keratin filament organisation so that difference in staining reflects differences in the ability of our antibodies to detect the keratins rather than differences in the amount of keratin.

We did in fact try using ICRF antibodies, but found that they did not work as well as the commercial ones on paraffin sections. An advantage of using commercial monoclonal antibodies is that they are well characterised and should be available to any laboratory wishing to compare results. Nevertheless, careful controls are required. We used DAB alone, omission of one reagent, use of non-immune serum, and comparison with other antisera. It is still possible that differences between cancers and normal tissue were largely owing to differences between paraffin blocks and frozen section material. In this respect we were fortunate to have cases that had both types of material and the staining was comparable (date not shown).

Additionally, all CIN3 and invasive cancer material in this paper were from paraffin blocks and both keratins 10 and 17 distinguished between them. Similarly, the results of tables 2 and 4 were largely unchanged when restricted to paraffin blocks (data not shown).

We suggest that the demonstration of cytokeratin profiles for 17 and 10 would be a helpful addition to the routine histological stains used by pathologists to improve the uniformity and prognostic value of their cervical reports. We hope, in the future, to investigate the use of $\mathrm{K} 17$ and K10 in cervical smears in the hope of supporting this suggested prognostic link.

This work was supported by the Imperial Cancer Research Fund

1 Smedts F, Ramaekers F, Link M, et al. Detection of keratin subtypes in routinely processed cervical tissue: implications for tumour classification and the study of cervix cancer aetiology. Virchows Arch 1994;425:145-55.

2 Anderson MC, Jordan JA, Morse AR, et al. A text and atlas of integrated colposcopy, 2nd ed. London: Chapman and Hall, 1996:1-37.

3 Anderson MC. The cervix, excluding cancer. In: Symmers WStC, ed. Systemic pathology, 3rd ed, vol 6. Edinburgh: Churchill Livingstone, 1991:47-65.

4 Moll R, Franke WW, Schiller DL, et al. The catalog of human cytokeratins: patterns of expression in normal epithelia, tumors and cultured cells. Cell 1982;31:11-21.

5 Lane EB, Alexander CM. Use of keratin antibodies in tumor diagnosis. Semin Cancer Biol 1990;1:165-79.

6 Maddox P, Szarewski A, Dyson J, et al. Cytokeratin expression and acetowhite change in cervical epithelium. If Clin Pathol 1994;47:15-17.

7 Smedts F, Raemaekers F, Robben H, et al. Changing patterns of keratin expression during progression of cervical intraepithelial neoplasia. Am f Pathol 1990;136:657-68.

8 Smedts F, Ramaekers F, Troyanovsky S, et al. Basal-cell keratins in cervical reserve cells and a comparison to their expression in cervical intraepithelial neoplasia. Am 7 Pathol 1992;140:601-12.

9 Smedts F, Raemaekers F, Troyanovsky S, et al. Keratin expression in cervical cancer. Am F Pathol 1992;141:497511.

10 Proby CM, Churchill L, Purkis PE, et al. Keratin 17 expression as a marker for epithelial transformation in viral warts. Am f Pathol 1993;143:1667-78.

11 Stasiak PC, Purkis PE, Leigh IM, et al. Keratin 19: predicted amino acid sequence and broad tissue distribution suggest it evolved from keratinocyte keratins. $\mathcal{F}$ Invest Dermatol 1989;92:707-16.

12 Gigi-Leitner O, Geiger B, Levy R, et al. Cytokeratin expression in squamous metaplasia of the human uterine cervix. Differentiation 1986;31:191-205.

13 Shi S, Key M. Antigen retrieval in formalin-fixed, paraffinembedded tissue: an enhancement method for IHC based on microwave oven heating of tissue sections. F Histochem Cytochem 1991;39:741-8.

14 Hamilton PW, Allen DC. Morphometry in histology. $\mathcal{f}$ Pathol 1995; 175:369-79.

15 Altman DG Practice statistics for medical research. London: Chapman and Hall, 1992:213-17; 351-8.

16 Markey AC, Lane EB, Churchill LJ, et al. Expression of simple epithelial K8 an K18 in epidermal neoplasia. $\mathcal{f}$ Invest Dermatol 1991;97:763-70.

17 Dallenbach-Hellweg G, Lang G. Immunohistochemical studies on uterine tumors. 1. Invasive squamous-cell carcinomas of the cervix and their precursors. Pathol Res Prac 1991;187:36-43.

18 Weiss RA, Eichner R, Sun T-T. Monoclonal antibody analysis of keratin expression in epidermal diseases; a 48and 56-kdalton keratin as molecular markers for hyperproliferative keratinocytes. I Cell Biol 1984;98:1397-406.

19 Blumenberg M, Jiang CK, Freedberg IM, et al. Cytokines and growth factors regulate keratin gene expression in wound healing and other pathological processes. $f$ Cell Biochem 1993;(suppl)17e:131.

20 Stafl A. Cervicography: a new method for cervical cancer detection. Am F Obstet Gynecol 1981;139:815-25. 